\title{
Scheduling Constraints and Interference Graph Properties for Graph-based Interference Coordination in Cellular OFDMA Networks
}

\author{
Marc C. Necker \\ Institute of Communication Networks and Computer Engineering, \\ University of Stuttgart, Pfaffenwaldring 47, D-70569 Stuttgart, Germany \\ Email: marc.necker@ikr.uni-stuttgart.de
}

\begin{abstract}
The currently emerging 802.16e (WiMAX) and 3GPP Long Term Evolution (LTE) cellular systems are based on Orthogonal Frequency Division Multiple Access (OFDMA). OFDMA suffers from heavy inter-cell interference if neighboring base stations use the same frequency range. One approach to solve this issue is the application of beamforming antennas in combination with interference coordination (IFCO) mechanisms between base stations. In this paper, we trace the problem of IFCO back to the graph coloring problem and investigate the achievable resource utilization of the interference coordinated system. We develop a heuristic that allows the combination of arbitrary scheduling algorithms with the IFCO mechanism. This allows an efficient utilization of the radio system's frequency resources while still obeying scheduling constraints, such as Quality of Service requirements. Finally, we study the tradeoff between fairness and the total system throughput.
\end{abstract}

Index Terms-OFDMA, 802.16e, WiMAX, 3GPP LTE, interference coordination, beamforming, graph coloring, scheduling

\section{INTRODUCTION}

Several emerging standards for broadband cellular communication are based on OFDMA. In particular, 802.16e (mobile WiMAX) and future 3GPP LTE cellular systems will offer high-speed packet switched services for a variety of applications. In OFDMA, the different users are multiplexed in time and frequency based on the underlying OFDM system, which basically corresponds to a combination of Frequency and Time Division Multiple Access (FDMA and TDMA). A major problem in FDMA/TDMA systems is inter-cell interference created by neighboring cells when using the same frequency band. This may lead to severe performance degradation or connection loss especially in the border areas of cells.

Classical FDMA/TDMA systems, such as GSM, solve this problem by avoiding the reuse of the same frequency range in neighboring cells. This reduces the utilization of scarce frequency resources. Instead, it is desirable to reuse the full frequency spectrum in every cell and mitigate inter-cellular interference by other means. One promising approach is interference coordination (IFCO), where neighboring base stations coordinate their transmissions in order to minimize interference. This is particularly effective when combined with beamforming antennas, which additionally allow the exploitation of Space-Division Multiplexing (SDM).

In [1], we presented a global IFCO algorithm for cellular OFDMA networks based on an interference graph. This graph represents critical interference relations among mobile terminals. A simple but efficient heuristic was used to solve the resource assignment problem, which required an omniscient device will full system knowledge. Even though this is not feasible in a real system, it provides valuable insight on the impact of important system properties and also yields an upper bound for the achievable performance. Furthermore, we developed two different distributed IFCO schemes in [2] and [3], where the interference graph is built in a central coordinator after collecting state information from all base stations. The interference graph is thus a powerful tool that can be used as a basis for present and future interference coordination algorithms.

In this paper, we extend our research from [1] and first study the basic properties of the interference graph. We then solve the resource assignment problem by tracing it back to the graph-coloring problem. By using a tabu search algorithm, we obtain near-optimal colorings of the interference graph, which allows us to draw conclusions on the resource utilization in the system. Subsequently, we study the impact of system constraints and scheduling on the resource assignment process. Finally, we present a heuristic that allows to trade off scheduling and fairness requirements with the total throughput.

This paper is an extension of a previous conference version from IEEE Broadnets 2007 [4]. It is structured as follows. After an overview of related work in section II, Section III introduces the cellular scenario. Section IV defines the interference graph and studies its properties. Subsequently, section V studies the pure graph coloring problem, and section VI the system imposed constraints and fairness issues. Finally, section VII concludes the paper.

\section{RELATED WORK}

\section{A. Classification}

Within the 3GPP LTE standardization, IFCO schemes are classified with respect to their time-scale of operation into static schemes, semi-static schemes, and dynamic schemes [5]. Static IFCO is done during the network planning process, for example by an optimized frequency planning in the network. They operate in the time-scale of days or longer. Semi-static schemes can be sub-divided into three sub-classes. First, self-configured coordination schemes are basically a self-optimizing version of static schemes. Second, cell load adaptive coordination requires load estimates of the cells and operates on the level of minutes. Third, user load adaptive coordination takes into account the instantaneous traffic demand, which varies faster than the cell load. Therefore, they operate 
on a time scale in the order of seconds or slightly below [5] or several hundred milliseconds [6]. Metrics for the traffic demand can for example be the buffer occupancy or the QoS profile of a particular traffic flow. Finally, fully dynamic IFCO schemes can instantly adapt to changing network conditions, such as changing traffic or load distributions. Their time scale of operation is in the order of one or only a few MAC frames.

With respect to the degree of distribution, four classes can be distinguished. Global schemes require an omniscient central entity that can capture the system state instantly and distribute scheduling decisions to all network nodes on a frame level. They allow to study basic system properties and tradeoffs and may serve as a basis for the development of more practical schemes. Distributed schemes require a central entity, but are designed to cope with real-world signaling delays and loads. Decentralized schemes operate without a central entity, but allow base stations to exchange information. Finally, local schemes get along without direct communication among base stations and operate only on local state information.

\section{B. Selected approaches from literature}

In circuit switched networks, techniques for Dynamic Channel Assignment (DCA) have been investigated as early as 1971 by Cox and Reudink [7]. Compared to classical frequency reuse with a static assignment of channels to cell sectors, DCA allows to dynamically re-assign channels from one cell to another. A second basic scheme besides DCA is Borrowing Channel Assignment (BCA), which has been proposed by Engel in [8], [9]. In contrast to DCA where all cell sectors may principally use all channels, BCA begins with a fixed channel assignment and then allows a fully loaded cell sector to borrow unused channels from one of its neighbor sectors.

Another popular technique is Reuse Partitioning. It is a form of static planning and aims at increasing the capacity by using different frequency reuse factors for certain mobile terminals. It was first introduced by Halpern in 1983 [10] under the name of reuse partitioning and re-invented in the late 1990ies as intelligent underlay-overlay [11], [12], [13], [14]. Within the activities of the WiMAX forum and 3GPP LTE standardization, it is known as Fractional Frequency Reuse (FFR) (e.g., [15]). While reuse partitioning and intelligent underlay-overlay stem from the era of circuit switched networks, the term FFR was coined in combination with packet switched networks.

A generalization of FFR was studied by Bonald et al. in [16], [17]. The authors define arbitrary geographic regions within a cell area, where in each region a terminal is served with a certain transmission profile. A transmission profile corresponds to a particular combination of active transceivers. Optimal region boundaries are then derived. The authors present numerical examples for a two-cell and a three-cell network, and for infinitely large linear and hexagonal networks. Liu and Virtamo present a very similar study in [18], taking further into account an arbitrary geographic traffic distribution.

A dynamic local coordination scheme was proposed by Sternad et al. in [19]. They coordinate the scheduling among cell sectors of the same base station and mitigate the interference from neighboring base stations by additionally applying reuse partitioning. Altogether, the authors achieve an effective frequency reuse of $1.5-2$ with an average SIR of $16 \mathrm{~dB}$ within the cell. In [20], we performed a comprehensive performance evaluation based on this and a related scheme.

A semi-static and decentralized IFCO scheme was proposed by Alcatel-Lucent in [21], which was further detailed and evaluated in [22], [23]. It is particularly useful for cell load balancing and for leveling out spatial concentrations of mobile terminals [22]. If a base station cannot serve all terminals in a cell sector at their required minimum bit rate, it sends a request to one or more interfering base station. These base stations may respond with a grant, increasing the capacity in the requesting cell sector.

In [24], [25], [26], Nortel proposes Adaptive Fractional Frequency Reuse, another semi-static and decentralized IFCO scheme based on FFR and soft frequency reuse. Every cell sector is served by using one of four modes, which are more or less aggressive with respect to the frequency utilization. Mode 1 corresponds to a frequency reuse of 1 where the full frequency band is available at full power. On the other end, Mode 4 corresponds to a frequency reuse of 3 . Modes 2 and 3 define a soft-reuse scheme with different power levels for the soft-reuse areas. The base stations communicate with each other and may request the usage of a less aggressive mode from their neighbors if interference levels become too high. A base station may thereby not use a more aggressive mode than was requested by any of its neighbors.

In [27], [28] and related documents, Texas Instruments proposes a variation of FFR, where the size of the reuse partitions varies between the cells and is adapted by a central coordinator in a semi-static manner. This concept therefore constitutes a semi-static distributed IFCO scheme. Other distributed schemes have been proposed by Rahman and Yanikomeroglu in [29] or by $\mathrm{Li}$ and Liu in [30].

IFCO has also been an active research area in multi-hop and mobile ad hoc networking for a longer time. In [31], the authors consider the possibility of beamforming in a multi-hop wireless network and study a MAC protocol which is capable of blocking the transmissions of the strongest interferers. In [32], the authors coordinate broadcasts in a multi-hop wireless network by means of a sequential graph coloring heuristic. In [33], the coordination of transmissions in a wireless ad-hoc network is considered. The interference conditions are evaluated by an omnipotent central entity with full system state information, which is able to schedule the data transmissions of the individual nodes on the MAC-frame level. This is done based on a conflict graph, which represents critical interference relations in-between the network nodes. The problem was traced back to the graph coloring problem for example in [34]. In [35], the throughput capacity of a wireless multi-hop network was calculated with the help of a very similar schedule graph, which is derived from physical layer properties of the network. 


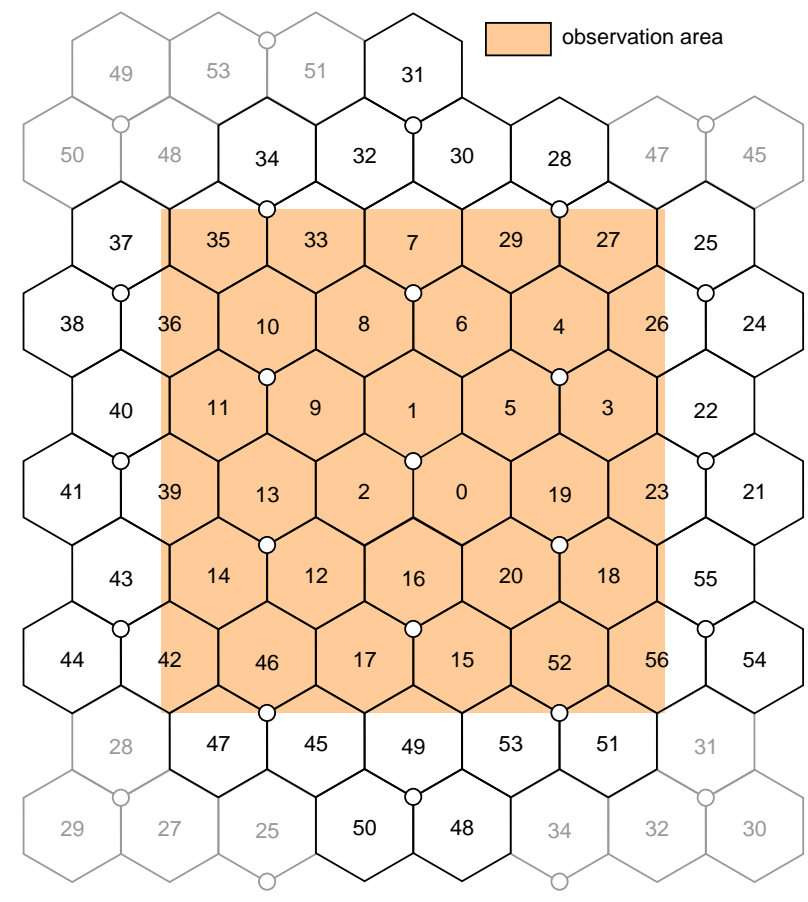

Figure 1. Hexagonal cell layout with wrap-around

\section{SYSTEM MODEL}

\section{A. Simulation scenario}

The simulation scenario was based on the recommendations of the 3GPP in [36], the Next Generation Mobile Networks alliance [37], and the WiMAX forum [38]. We therefore consider a hexagonal cell layout comprising 19 base stations at a distance of $d_{B S}=1400 \mathrm{~m}$ with wrap-around. This is shown in Fig. 1. The wrap-around property infinitely repeats the 19 base stations in the xy-plane. Therefore, there is no distinct center cell and all cells are equal. This leads to a completely symmetric and balanced scenario. Every base station has three $120^{\circ}$ cell sectors, where each sector is served by one transceiver. The transceivers are equipped with linear array beamforming antennas with four antenna elements and radiation patterns according to [1]. They can be steered towards each terminal with an accuracy of 1 degree, and all terminals can be tracked ideally. Throughout the paper, only the downlink direction is considered. However, similar considerations can be made for the uplink.

\section{B. Metrics}

The performance evaluation is done based on direct and indirect metrics. Direct metrics are the aggregate sector throughput, which relates to the overall spectral efficiency of the system, and the cell edge throughput, which takes into account the fairness towards the cell edge terminals. The latter can be captured by measuring the 5\% throughput quantile [36]. Indirect metrics are the Signal-to-Interference-Ratio (SIR) and the Signal-to-Interference-Noise-Ratio (SINR). Additionally, the resource utilization $\rho$ is of interest, which can directly be related to an effective frequency reuse factor. For example, in a frequency reuse 3 system, the resource utilization is $\rho=1 / 3$. We further define a practical indirect metric to measure fairness in Section VI-C.

\section{Simulation model for indirect metrics}

In order to measure indirect metrics, we perform MonteCarlo simulations, where $N$ mobile terminals per cell sector are placed at random positions in each drop. The simulation model is based on an 802.16e-system [39] with a total available bandwidth of $10 \mathrm{MHz}$ and a MAC-frame length of $5 \mathrm{~ms}$. All cells were assumed to be synchronized on a frame level. We focus on the downlink direction and consider the downlink Partial Use of Sub-Carriers (PUSC) and band Adaptive Modulation and Coding (band AMC) zones.

\section{Simulation model for direct metrics}

Throughput results are captured at the IP-level. Instead of Monte-Carlo simulations, a frame-level simulation with random direction mobility of the mobile terminals at a velocity of $v=30 \mathrm{~km} / \mathrm{h}$ similar to [1] is conducted. Throughput results were obtained for the band AMC $2 \times 3$ mode, which defines slots of 16 data subcarriers by 3 OFDM-symbols. This is illustrated in the left part of Fig. 2. A slot corresponds to the resource assignment granularity for a particular mobile terminal. The AMC zone can therefore be abstracted by the two-dimensional resource field shown in the right part of Fig. 2. The band AMC zone was assumed to consist of 9 OFDM-symbols, corresponding to a total number of $48 \cdot 3$ available slots. All relevant MAC-mechanisms, such as fragmentation, Automatic Repeat Request (ARQ) and Hybrid ARQ (HARQ) where modeled in detail. Adaptive Modulation and Coding was applied ranging from QPSK 1/2 to 64QAM $3 / 4$, which results in a theoretical maximum raw data rate of about $6.2 \mathrm{Mbps}$ within the band AMC zone. The burst profile management was based on an exponential average of a
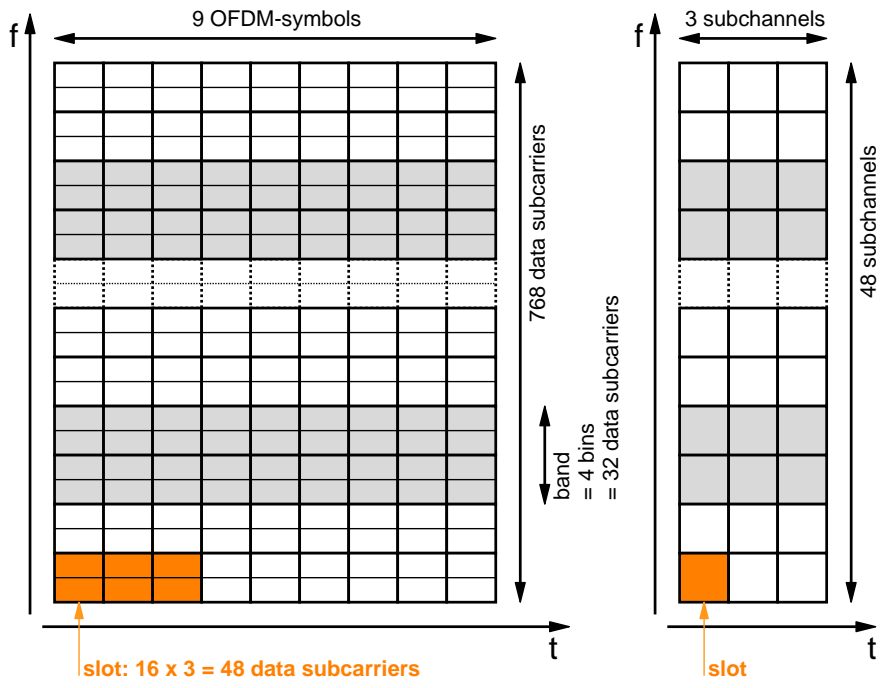

Figure 2. Illustration of the band AMC $2 \times 3$ mode 
mobile terminal's SINR conditions. The MCS was selected for a target burst loss probability of $10 \%$, which is a commonly used value in packet switched cellular networks. Note that due to the beamforming antennas, the burst profile management is impaired by strong interference fluctuations, and hence, the actual burst loss probability may be significantly larger than $10 \%$.

\section{INTERFERENCE COORDINATION AND RESOURCE ASSIGNMENT}

\section{A. General procedure}

Each sector has its own set of resources (see Fig. 2), which need to be assigned to the terminals in every MAC-frame. In order to realize the coordination of cell sectors, we divide the scheduling process into two parts:

1) Creation of interference graph: In this step, a graph is created based on the interference relations among all terminals. Its vertices represent the mobile terminals and its edges represent critical interference relations in-between them. In particular, terminals which are connected must not be served using the same set of resources. Figure 3 shows an example of an interference graph.

This interference graph is similar to the conflict graph used in [33] regarding its semantics. However, it is constructed differently in our case, since terminals are only receiving but not transmitting any data.

2) Resource assignment: In this second step, resources are assigned to the terminals while taking into account the constraints of the interference graph. In section $\mathrm{V}$, the resource assignment problem is treated by means of graph coloring algorithms. In section VI, we additionally take into account constraints of the 802.16e transmission system and present a heuristic to assign resources in combination with an arbitrary scheduling algorithm.

In the following subsections, we will describe the construction of the interference graph and study its basic properties.

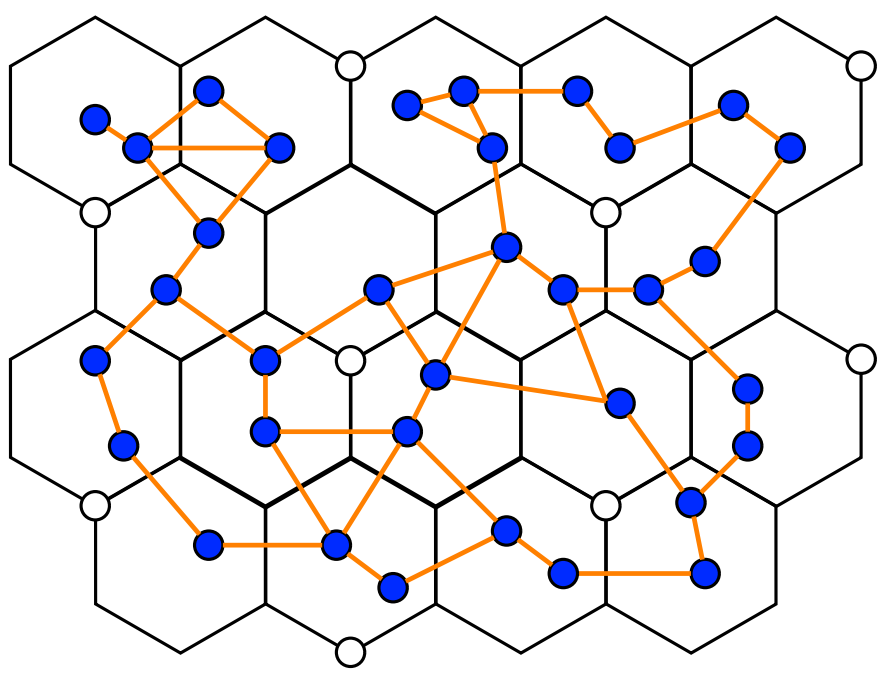

Figure 3. Example of interference graph

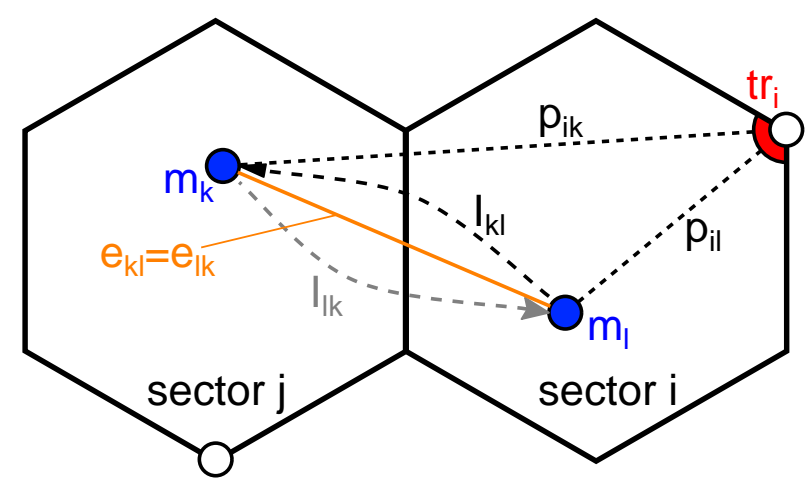

Figure 4. Creation of interference graph

Note that while the above description focuses on a global IFCO scheme with an omniscient entity, many of the following considerations are also valid for a distributed IFCO algorithm that applies an interference graph, as for example the algorithm proposed in [3].

\section{B. Construction of the interference graph}

In this section, we briefly review the creation of the interference graph as we introduced it in [1]. We construct the interference graph by evaluating the interference that a transmission to one mobile terminal causes to any other terminal. For each terminal, we first calculate the total interference and then block the largest interferers from using the same set of resources by establishing a relation in the interference graph. This is done such that a desired minimum SIR $D_{S}$ is achieved.

Let $m_{k}$ and $m_{l}$ be two mobile terminals in different cell sectors, as illustrated in Fig. 4. $t r_{i}$ denotes the transceiver serving cell sector $i . p_{i k}$ describes the path loss from transceiver $t r_{i}$ to terminal $m_{k}$, including shadowing. We further introduce the function $G_{i}(l, k) .{ }^{1}$ It describes the gain of the sector $i$ beamforming antenna towards terminal $m_{k}$ when the array is directed towards terminal $m_{l} . e_{k l} \in\{0,1\}$ are the elements of the interference graph's adjacency matrix $E$, indicating an interference relation between terminals $m_{k}$ and $m_{l}$ if $e_{k l}=1$.

In a first step, we calculate the interference $I_{k l}$ which a transmission to mobile $m_{l}$ in sector $i$ would cause to mobile $m_{k}$ in sector $j$, where $i \neq j$ :

$$
I_{k l}=p_{i k} G_{i}(l, k) P_{l},
$$

where $P_{l}$ is the transmission power of transceiver $i$ towards terminal $m_{l}$. For each terminal $m_{k}$, we collect all interference relations in the set $W_{k}$ :

$$
W_{k}=\left\{I_{k l}, \quad \forall l \neq k,\left|c_{l}-c_{k}\right| \leq d_{i c}\right\} .
$$

$c_{l}$ are the geographic coordinates of the transceiver serving the cell sector where terminal $m_{l}$ is located in. The coordination diameter $d_{i c}$ then denotes the maximum distance which two base stations may have in order to still be coordinated.

We then keep removing the largest interferer from $W_{k}$ until

${ }^{1} G_{i}$ corresponds to all possible gain patterns of the beamforming antenna, which were obtained with a separate MatLab program from standard beamforming signal processing algorithms. 

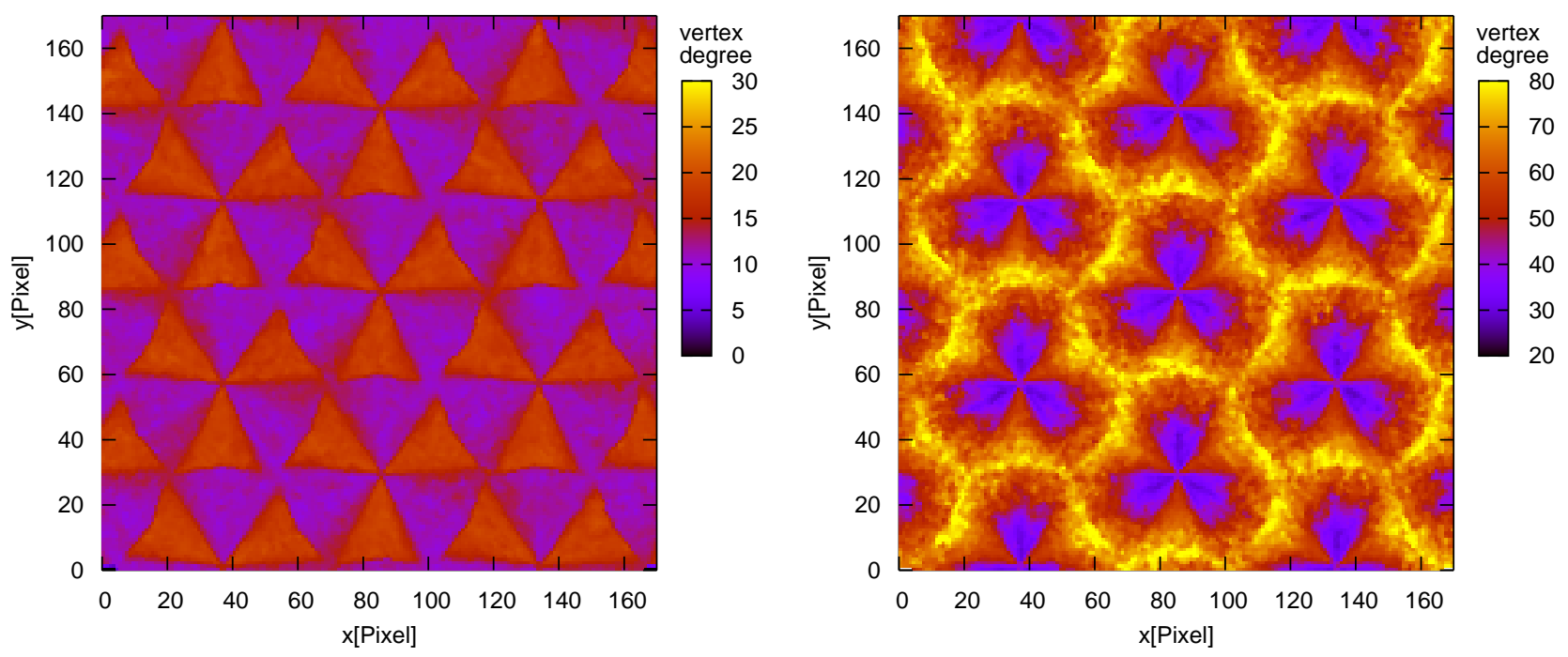

Figure 5. Vertex degree depending on terminal location for $d_{i c}=0$ (left) and $d_{i c}=1$ (right), $N=8, D_{S}=15 \mathrm{~dB}$

the worst-case SIR for terminal $m_{k}$ rises above a given desired SIR threshold $D_{S}$ :

$$
\mathrm{SIR}_{k}=\frac{S_{k}}{\sum_{I_{k l} \in W_{k}} I_{k l}} \geq D_{S} .
$$

$S_{k}$ is the received signal strength of terminal $m_{k}$ if it is served:

$$
S_{k}=p_{j k} G_{j}(k, k) P_{k} \text {. }
$$

The edges $e_{k l}$ of the interference graph then follow as:

$$
e_{k l}=\left\{\begin{array}{ll}
0 & \text { if } I_{k l} \in W_{k} \wedge I_{l k} \in W_{l} \\
1 & \text { otherwise }
\end{array} .\right.
$$

Equation (5) sets an interference relation $e_{k l}$ if terminal $m_{k}$ causes interference to terminal $m_{l}$, or vice versa. This is necessary since in both cases the usage of the same set of resources must be avoided. This results in a non-directional interference graph, i.e., $E$ is symmetric.

Finally, all mobile terminals within a sector must be assigned disjoint resources. Hence, $e_{k l}=1$ if $m_{k}$ and $m_{l}$ belong to the same sector, which leads to a modification of eq. (5):

$$
e_{k l}= \begin{cases}0 & \text { if } I_{k l} \in W_{k} \wedge I_{l k} \in W_{l} \wedge C\left(m_{l}\right) \neq C\left(m_{k}\right) \\ 1 & \text { otherwise }\end{cases}
$$

where $C\left(m_{l}\right)$ denotes the cell sector serving mobile $m_{l}$.

\section{Characteristics of the interference graph}

The degree of each vertex describes the amount of critical interference relations for each terminal. In areas with good reception conditions the vertex degree is likely to be low, while it is expected to be high in areas with bad reception conditions. Furthermore, the vertex degree depends on the number of mobile terminals $N$ and on the coordination diameter $d_{i c}$. For $d_{i c} \geq d_{B S}$, a global graph is generated. It can be used within a distributed coordination scheme as we did in [2] and [3], or it can be used as the basis of a global coordination scheme. A local graph is obtained with $d_{i c}=0$. No communication between base stations is required when using a local graph, as coordination takes place only among the cell sectors of every base station. Such a coordination scheme is well implementable, as it uses only local state information in every base station. It was shown in [40] that the a locally coordinated network cannot match the performance of a globally coordinated network. Still, it achieves an aggregate performance gain of about $25 \%$ with respect to the aggregate throughput and of about $35 \%$ with respect to the cell edge throughput compared to an uncoordinated frequency reuse 1 system.

Figure 5 illustrates the vertex degree within the observation area (as defined in Fig. 1) for $D_{S}=15 \mathrm{~dB}$ and $N=8$. For $d_{i c}=0$, the vertex degree is relatively low throughout the area. A high vertex degree can be found in the border areas between cell sectors of the same base station. They suffer from interference due to the relatively large width of the antenna beam and its significant side-lobes when the beam is directed towards the left or right cell edge. The vertex degree is low in the boresight direction of the cell sectors, which can be well covered by the beamforming antennas and which receive relatively small interference from neighboring sectors. In contrast, the vertex degree behaves essentially different for $d_{i c}=1$. Now, we can additionally observe a high vertex degree in the border areas between base stations and between cell sectors, since the interference graph now also reflects inter-cell interference from neighboring base stations.

\section{Resource Assignment by Graph Coloring}

\section{A. The graph coloring problem}

During the resource assignment step, the constraints of the interference graph have to be taken into account. As all 


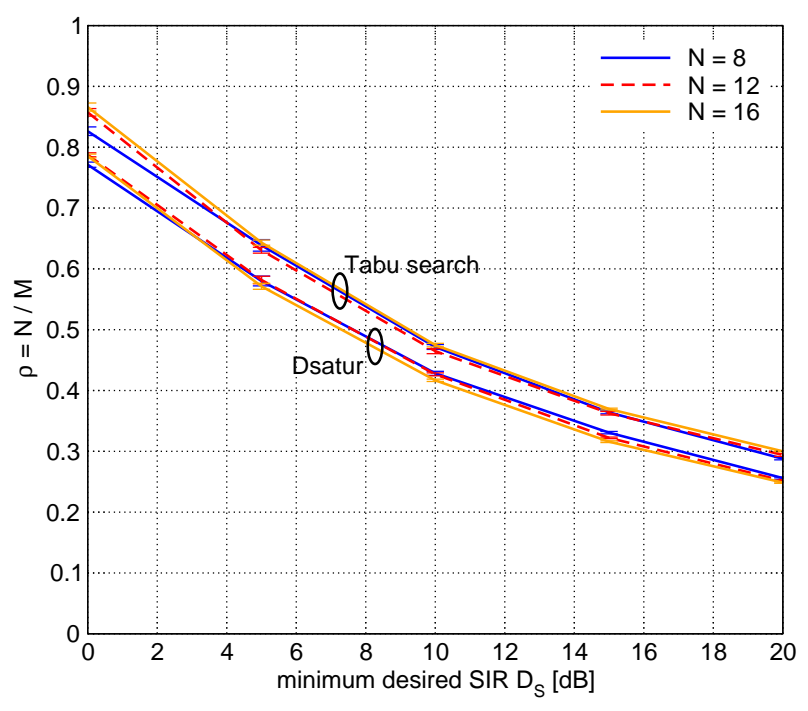

Figure 6. Resource utilization $\rho=N / M$ for different number of terminals $N, d_{i c}=1$

terminals which are connected in the interference graph must not be served by using the same set of resources, we have to find the minimum set of disjoint resources such that this requirement is met. This directly translates to the well-known graph coloring problem, in which the interference graph has to be colored such that no two connected vertices have the same color. The colors of the vertices correspond to particular resource sets, for example to disjoint sets of subchannels in the AMC- or PUSC-zone. Each set can then be used for the transmission towards a mobile terminal.

The number of colors $M$ which are needed for the coloring determines the utilization of transmission resources in the system. If $M$ colors are needed, $M$ disjoint resource sets have to be formed. In each cell sector, only $N$ of these resource sets will eventually be used, which implies a resource utilization of $\rho=N / M$. In our problem, the minimum clique size of the interference graph is equal to the number of mobile terminals $N$ per cell sector. Consequently, at least $N$ colors are needed. Most cases will require more colors, i.e., $M>N$, leading to a resource utilization below $100 \%$.

The graph coloring problem is NP hard. In order to solve the coloring problem, we apply the heuristic Dsatur [41] and a tabu search technique [42]. While tabu search usually finds a smaller set of colors than the Dsatur algorithm, it is computationally much more expensive.

\section{B. Resource utilization}

As the minimum desired SIR $D_{S}$ is increased, the average vertex degree of the interference graph increases. This leads to an increase in the number of used colors and to a reduction of the resource utilization $\rho$. This is illustrated in Fig. 6, which plots $\rho$ over $D_{S}$ for $d_{i c}=d_{B S}$. Shown are the results when applying Dsatur and tabu search, respectively. As expected, the resource utilization decreases if $D_{S}$ is increased, since more conflicts in the interference graph are produced

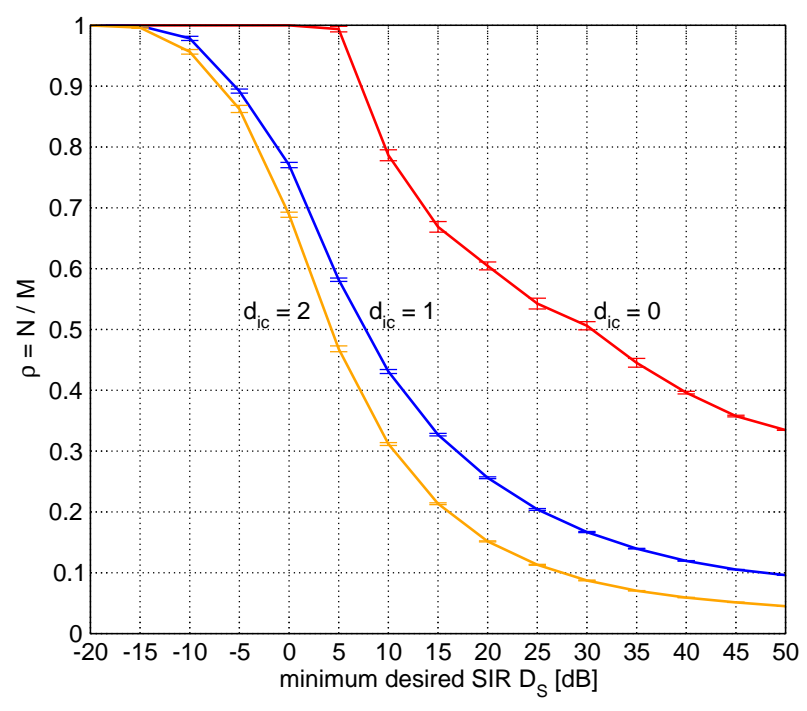

Figure 7. Resource utilization $\rho=N / M$ depending on $d_{i c}, N=8$, $D_{S}=15 \mathrm{~dB}, d_{i c}=1$

and more colors are needed. The general behavior of the resource utilization with Dsatur is similar to that with tabu search, although, the computationally much more expensive tabu search produces a significantly better resource utilization.

Despite these expected results, the relationship between the number of mobile terminals $M$ and the resource utilization $\rho$ is not obvious. If we increase the number of mobile terminals $N$ in each cell sector, we also expect an increase in the number of required colors $M$. However, this does not allow any general conclusion for the ratio $\rho=N / M$, since the increase in $N$ may compensate for the increase of $M$. In fact, Fig. 6 shows that an increase in the number of mobile terminals from $N=8$ to 12 or 16 leads to no significant changes in the resource utilization.

Figure 7 plots $\rho$ over $D_{S}$ for different coordination diameters $d_{i c}$. Due to the increased complexity of the interference graph and the higher vertex degree which became obvious in Fig. 5, the resource utilization drops significantly as $d_{i c}$ is increased from 0 to $d_{B S}$. Further increasing $d_{i c}$ only leads to a small decrease in the resource utilization since the second tier of base stations adds only a small amount of interference.

Figure 8 plots the pdf of the color indices, which are used for the graph coloring in average. For each individual coloring, the indices are sorted such that lower indices indicate more frequently used colors. The pdf reveals that the different colors are distributed unequally across the terminals. In particular, a small number of mobile terminals require extra colors in order solve the coloring problem. This decreases the resource utilization while at the same time it only supplies a small number of additional terminals. It is therefore worthwhile to consider the possibility of not serving all mobile terminals in each frame, but only a selection, possibly those terminals which are most favorable with respect to the graph coloring problem. In the following section, we will consider this issue 
among with certain other system imposed constraints.

\section{Resource Assignment in Practice}

\section{A. Impact of system constraints and QoS requirements}

The evaluation in the previous section provided a basic analysis of the interference coordination problem and demonstrated the impact of the number of mobile terminals and the desired minimum SIR on the achievable resource utilization. It was assumed that all terminals are served in each radio frame. This will not be the case in a real system for several reasons. First, each transmission towards a terminal generates signaling overhead due to the transmission of the downlink map information in $802.16 \mathrm{e}$ and connection related signaling messages in the uplink direction. Second, the available resources are not arbitrarily divisible, and each transmission requires a certain minimum amount of granted resources due to the minimum ARQ block size, which cannot be further fragmented.

Another important system aspect is fairness. Compared to terminals in the cell center area, terminals located at the cell border areas are penalized in two ways. First, they suffer from worse SINR conditions due to a higher path-loss and a larger inter-cell interference, which lead to a lower throughput. Consequently, a higher amount of transmission resources is required to achieve a certain data rate. Second, they produce a larger number of conflicts in the interference graph $(\mathrm{cmp}$. Fig. 5), which overproportionally increases the number of colors needed for the graph coloring. Both these facts make it expensive to serve terminals at the cell border areas. However, it is important to provide a certain Quality of Service (QoS) to each connection, leading to a tradeoff with the overall achievable sector throughput.

The graph coloring approach of section $\mathrm{V}$ treats all terminals equally, allowing each terminal to be served with an equal amount of transmission resources. This eventually corresponds to a Round Robin Scheduling of all terminals. On the one

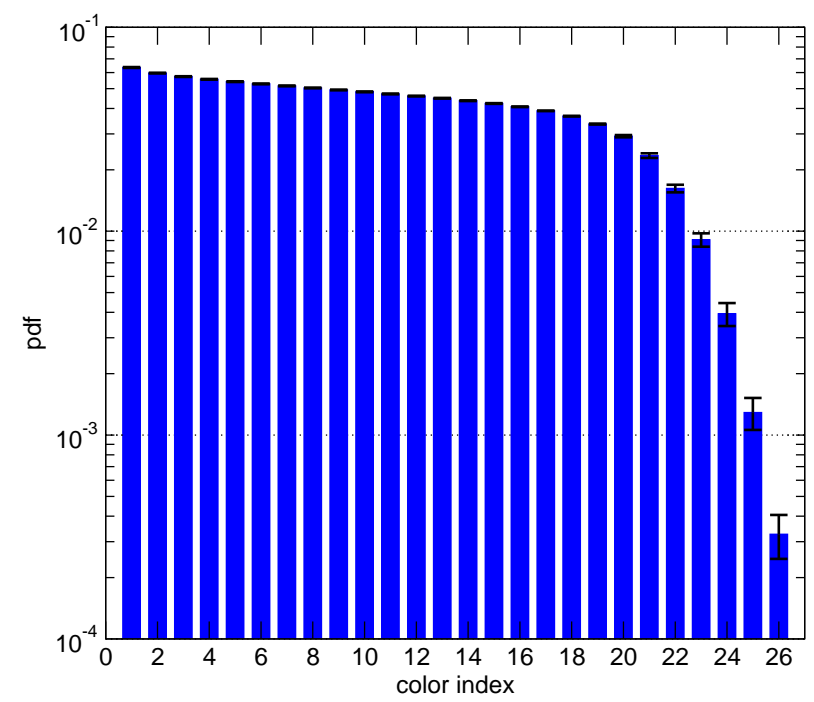

Figure 8. pdf of used color indices, $N=8, D_{S}=15 \mathrm{~dB}, d_{i c}=1$

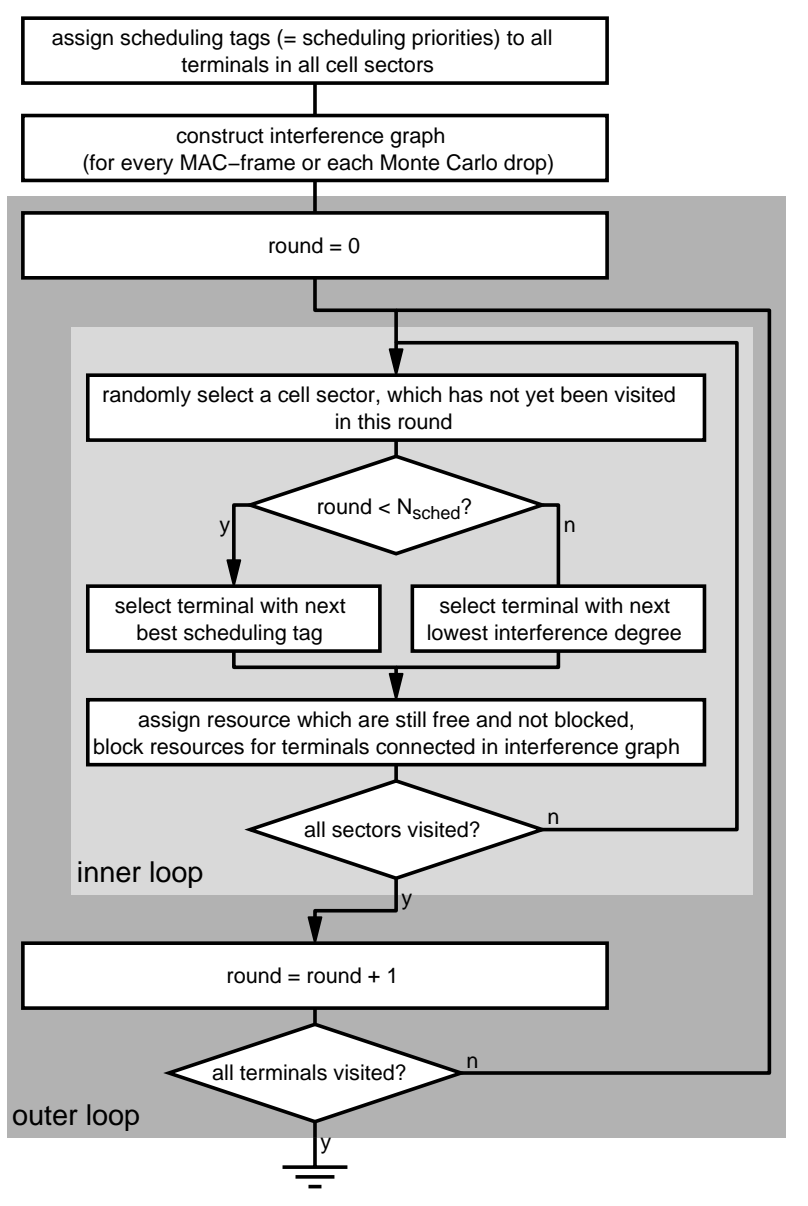

Figure 9. Heuristic for scheduling and resource assignment in every frame

hand, the equal treatment of terminals even in the cell border areas results in a rather low resource utilization. On the other hand, it is desirable to provide arbitrary QoS to each connection by means of a scheduling algorithm even in the cell border areas. In the following sections, we will investigate this tradeoff between the resource utilization and the fairness towards all terminals.

\section{B. Heuristic for combined scheduling and resource assign- ment}

Based on the previous considerations we present a heuristic which combines an arbitrary scheduling algorithm with the graph coloring problem. As discussed in the previous section, only a certain number $N_{\text {srvd }}$ of mobile terminals will be served in each frame. This allows to choose $N_{s r v d}$ terminals out of the $N$ terminals in each cell sector according to a certain criteria. This may either be determined by a particular scheduling algorithm, but it may also be based on which terminals are best suitable to solve the graph coloring problem with a minimum number of colors. The latter case is related (but not identical) to the clique coloring problem for $N_{\text {srvd }}=2$. A simple heuristic approach is to serve the terminals with the lowest vertex degree, since they produce the fewest conflicts.

In order to evaluate the impact of the scheduling algorithm, 

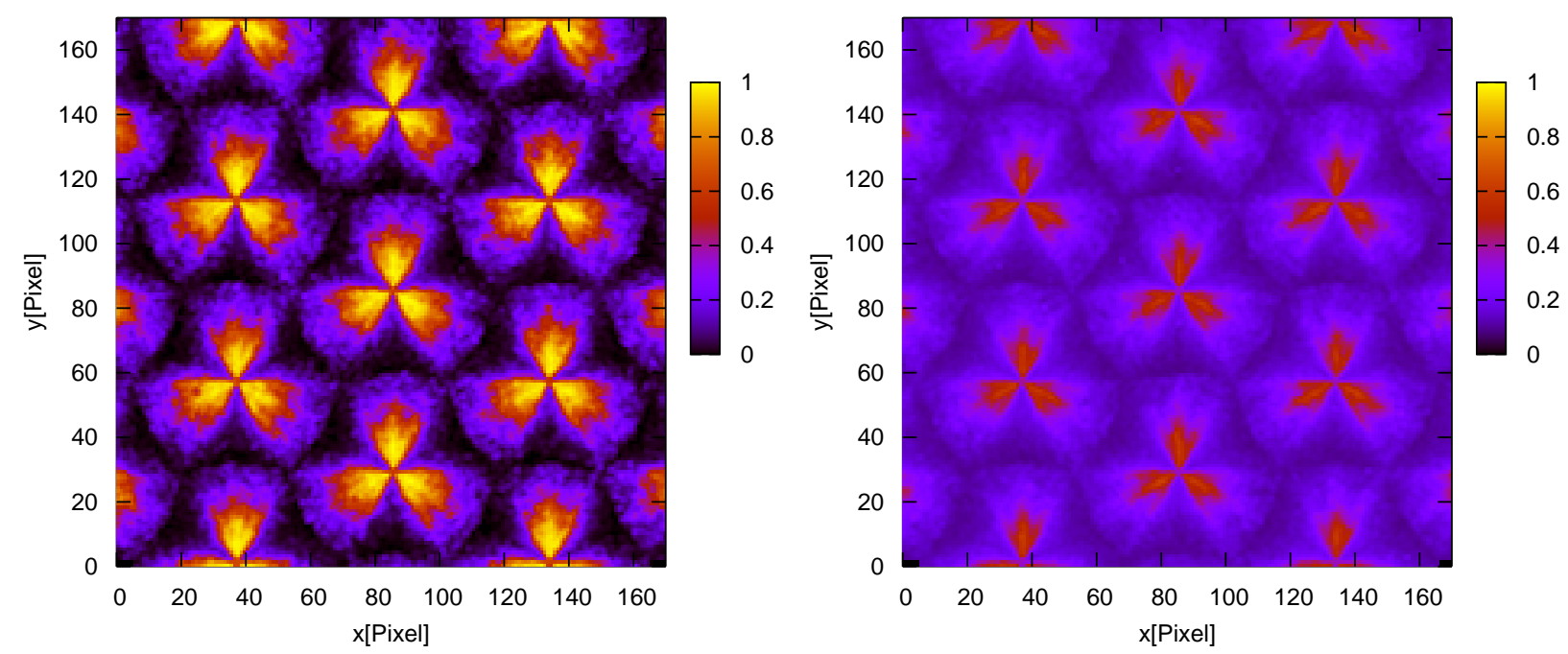

Figure 11. Fraction of radio frames $f(\vec{x})$ assigned for terminals at a particular location for $N=8, D_{S}=15 \mathrm{~dB}, d_{i c}=1, N_{s r v d}=4, N_{s c h e d}=0$ (left) and $N_{\text {sched }}=4$ (right)

we will assign resources to $N_{\text {sched }}$ terminals based on the scheduler's decision, and to the remaining $N_{\text {srvd }}-N_{\text {sched }}$ terminals based on their vertex degree. The flow-chart of the heuristic algorithm is shown in Fig. 9. The inner loop traverses all cell sectors in a random order and assigns resources to one terminal within every sector. The outer loop counts the number of rounds, where one round corresponds to the assignment of resources to one terminal in each cell sector. In the inner loop, the terminal to be served is selected based on the index of the current round. This is done such that in the first $N_{\text {sched }}$ rounds the terminal is selected according to the scheduler's

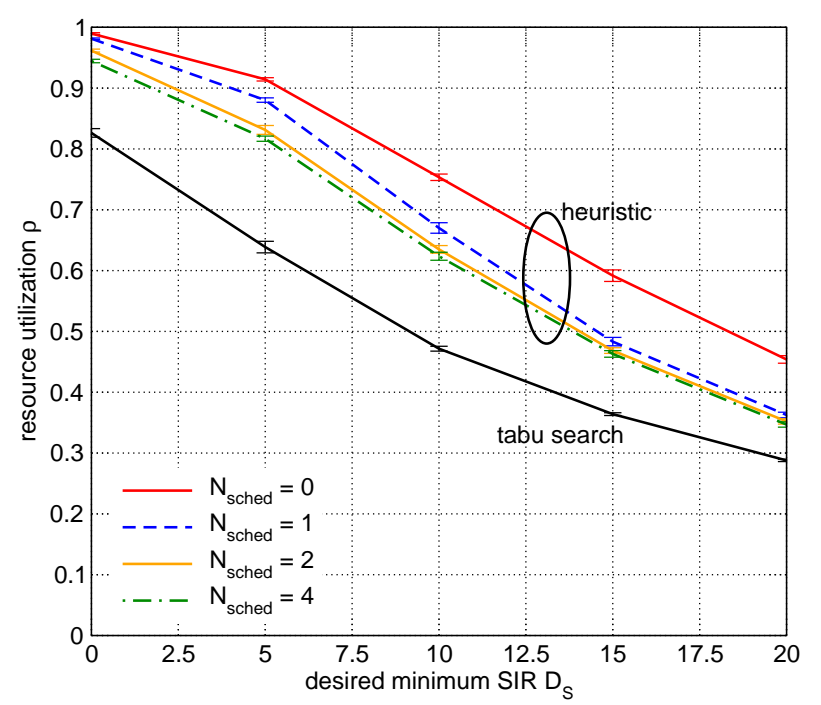

Figure 10. Resource Utilization $\rho$ achieved using the heuristic with different values of $N_{\text {sched }}$ and using the graph coloring with tabu search, $N=8$, $d_{i c}=1, N_{\text {srvd }}=4$ decision, and in all remaining rounds the terminal is selected based on its vertex degree. Whenever resources are assigned to a mobile terminal, these resources are blocked for all other mobile terminals, which are connected to it in the interference graph. Resources are assigned by dividing the AMC zone into $N_{\text {srvd }}$ equally sized disjoint sets of subchannels and assigning the first free and non-blocked set to the current terminal. The algorithm terminates once it has tried to assign resources to all mobile terminals.

\section{Impact of scheduling}

As an example, a Random scheduling mechanism is used. The Random scheduler assigns the highest scheduling priority to each of the $N$ mobile terminals in a cell sector at least once within repeating periods of $N$ MAC-frames. Moreover, the terminal with the highest priority in frame $n$ becomes the mobile with the second highest priority in frame $n+1$, and so on. The considered Random scheduler therefore behaves very much like a Round Robin scheduler on short and long time scales.

Figure 10 plots the resource utilization $\rho$ over $D_{S}$ for different values of $N_{\text {sched }}$. If we set $N_{\text {sched }}$ to 0 , the scheduler has no influence. Instead, terminals with favorable interference properties will be served preferably. In particular, the system will give preference to terminals with a low vertex degree in the interference graph, which increases the resource utilization at the cost of fairness. As $N_{\text {sched }}$ is increased, more terminals are served based on the scheduler's decision. This leads to a decrease in the resource utilization. However, this increases the fairness, which is illustrated in Fig. 11 and 12.

In Fig. 11, the fraction of radio frames $f(\vec{x})$ in which resources are assigned to a particular terminal depending on its position in the observation area is plotted, where $\vec{x}$ denotes 


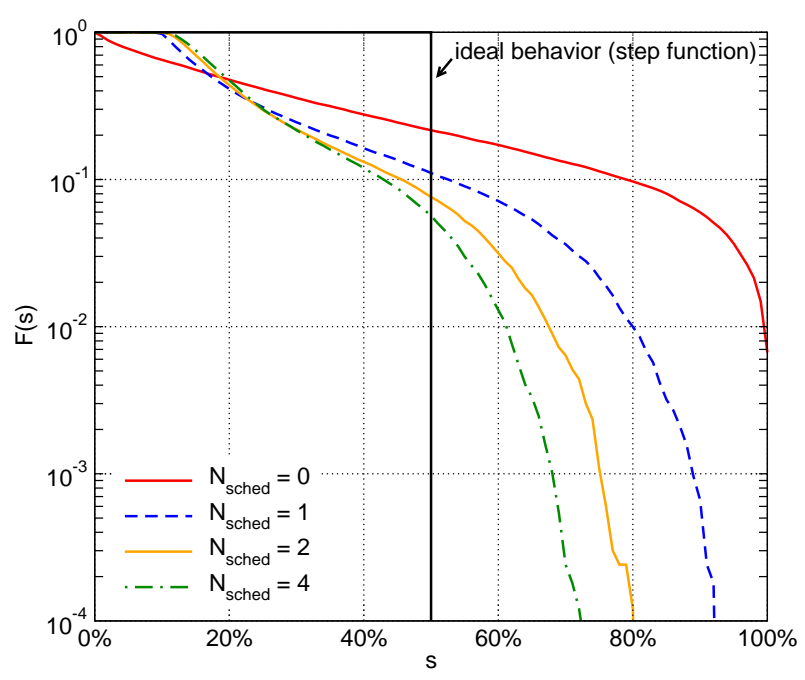

Figure 12. ccdf $F(s)$ of the fraction $s$ of radio frames $f(\vec{x})$ used at a particular position, $N=8, D_{S}=15 \mathrm{~dB}, d_{i c}=1, N_{\text {srvd }}=4$

the geographic position of the terminal. A value of $f(\vec{x})=0$ means that a mobile terminal at the respective position is never served, while a value of $f(\vec{x})=1$ means that it is served in every radio frame. For $N_{\text {sched }}=0$ we can see large areas at the cell border where mobile terminals are hardly ever served while in the center areas terminals are served in almost every frame. This implies that the coverage of the system is decreased. In contrast, for $N_{\text {sched }}=4$, the assignment of resources to terminals is much less dependent on their position. The still higher serving probability in the cell center can be traced back to the circumstance that these terminals can more easily fill resource gaps after all terminals have been served based on the scheduler's decision.

Further insight can be gained by looking at the distribution of $f(\vec{x})$. The complementary cumulative distribution function (ccdf) $F(s)$ is calculated as

$$
F(s)=P(f(\vec{x})>s) .
$$

The ccdf $F(s)$ is plotted in Figure 12. For $N_{\text {sched }}=0$, the ccdf indicates a significant probability of about $6 \%$ for $f(\vec{x})=0$, i.e., for no coverage. On the other hand, in $10 \%$ of all locations, mobile terminals receive resources in $80 \%$ of all radio frames. As $N_{\text {sched }}$ is increased, the coverage immediately increases to $100 \%$ of the cell sector area, and there are less areas where terminals are served excessively often. Ideally, this ccdf would be an inverted and shifted step function with the step at $s=\min \left(1, N_{\text {srvd }} / N\right)$, which would indicate an equal assignment of resources to terminals independent of their position.

\section{Throughput results}

Figure 13 shows the total IP-level sector throughput over $D_{S}$ for $N_{\text {srvd }}=4$ and different values of $N_{\text {sched }}$. As discussed in [1], the throughput shows a maximum for a particular $D_{S}$, which is due to the tradeoff of the resource utilization

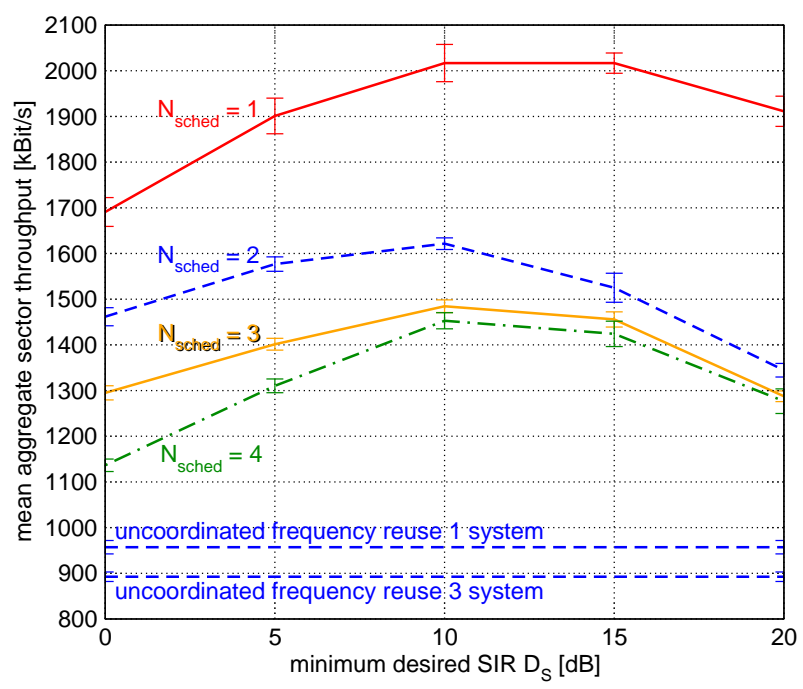

Figure 13. Total IP-level sector throughput, $N=8, D_{S}=15 \mathrm{~dB}$, $d_{i c}=1, N_{\text {srvd }}=4$

and the achievable SINR. For $N_{\text {sched }}=4$, the maximum total sector throughput is about $1453 \mathrm{kBit} / \mathrm{s}$, while for $N_{\text {sched }}=0$, it increases by almost $40 \%$ to about $2017 \mathrm{kBit} / \mathrm{s}$, however at the expense of the before discussed coverage loss. The chart also shows the results for uncoordinated reference systems with a frequency reuse of 1 and 3, respectively. These systems achieve an aggregate sector throughput of about $957 \mathrm{kBit} / \mathrm{s}$ and $892 \mathrm{kBit} / \mathrm{s}$, respectively. The throughput gain of the interference coordinated system is therefore significant and may be traded off against fairness by the parameter $N_{\text {sched }}$.

\section{CONCLUSION}

In this paper, we solved the problem of interference coordination in cellular OFDMA networks by means of graph coloring. We showed that some colors during the graph coloring are only used by a few mobile devices, thus decreasing the resource utilization. Further, the number of necessary colors proved to be independent of the number of mobile terminals in the considered range, which implies an equal resource utilization independent of the number of mobile terminals. We also presented a heuristic which allows the combination of arbitrary scheduling algorithms with interference coordination. This heuristic allows to trade off scheduler requirements, such as Fairness and QoS constraints with the total sector throughput. It was seen that the total sector throughput can be increased by almost $40 \%$ at the cost of fairness and coverage.

\section{ACKNOWLEDGMENTS}

This research was done in cooperation with Alcatel-Lucent Research and Innovation Department, Stuttgart. ${ }^{2}$ The author would like to thank Martin Köhn and Michael Scharf for their valuable input, and Joe Culberson for his graph coloring tools.

\footnotetext{
${ }^{2}$ Alcatel-Lucent Deutschland AG, Research \& Innovation, Holderäckerstr. 35, 70435 Stuttgart, Germany. Contact: Michael Tangemann (Michael.Tangemann@alcatel-lucent.de).
} 


\section{REFERENCES}

[1] M. C. Necker, "Towards frequency reuse 1 cellular FDM/TDM systems," in Proc. 9th ACM/IEEE International Symposium on Modeling, Analysis and Simulation of Wireless and Mobile Systems (MSWiM 2006), Torremolinos, Malaga, Spain, October 2006, pp. 338-346.

[2] —_, "Coordinated fractional frequency reuse," in Proc. 9th ACM/IEEE International Symposium on Modeling, Analysis and Simulation of Wireless and Mobile Systems (MSWiM 2007), Chania, Crete Island, Greece, October 2007, pp. 296-305.

[3] _ " "A graph-based scheme for distributed interference coordination in cellular OFDMA networks," in Proc. 67th IEEE Vehicular Technology Conference (VTC 2008-Spring), Singapore City, Singapore, May 2008, pp. $713-718$.

[4] 1 , "Integrated scheduling and interference coordination in cellular OFDMA networks," in Proc. IEEE 4th International Conference on Broadband Communications, Networks, and Systems (Broadnets 2007), Raleigh, NC, USA, September 2007.

[5] 3GPP TSG RAN WG1\#44 R1-060586, "Downlink and uplink intercell interference co-ordination/avoidance - impact on the specifications," Ericsson, NTT DoCoMo, Denver, USA, Tech. Rep., February 2006.

[6] 3GPP TSG RAN WG1 Ad Hoc on LTE R1-060135, "Interference mititgation by partial frequency reuse," Siemens, Helsinki, Finland, Tech. Rep., January 2006, ericsson.

[7] D. C. Cox and D. O. Reudink, "Dynamic channel assignment in high-capacity mobile communications systems," Bell System Technical Journal, vol. 50, no. 6, pp. 1833-1857, July-August 1971.

[8] J. Engel and M. Peritsky, "Statistically-optimum dynamic server assignment in systems with interfering servers," IEEE Transactions on Communications, vol. 21, no. 11, pp. 1287-1293, November 1973.

[9] — "Statistically-optimum dynamic server assignment in systems with interfering servers," IEEE Transactions on Vehicular Technology, vol. 22, no. 4, pp. 203-209, November 1973.

[10] S. Halpern, "Reuse partitioning in cellular systems," in Proc. 33rd IEEE Vehicular Technology Conference (VTC 1983), vol. 33, Toronto, ON, Canada, May 1983, pp. 322-327.

[11] Y. Ling, J. Elling, and T. Toftegaard, "Capacity of intelligent underlay and overlay network," in Proc. IEE Colloquium on Advanced TDMA Techniques and Applications (Digest No: 1996/234), London, UK, October 1996, pp. 5/1-5/9.

[12] V. Wille, S. Irons, and A. King, "Capacity increase in cellular radio networks using existing base station sites," in Proc. IEE Colloquium on Propagation Aspects of Future Mobile Systems (Digest No: 1996/220), London, UK, October 1996, pp. 4/1-4/8

[13] T. Nielsen, J. Wigard, and P. Mogensen, "On the capacity of a GSM frequency hopping network with intelligent underlay-overlay," in Proc. 47th IEEE Vehicular Technology Conference (VTC 1997), vol. 3, Phoenix, AZ, USA, May 1997, pp. 1867-1871.

[14] J. Wigard, T. Nielsen, P. Michaelsen, and P. Mogensen, "Improved intelligent underlay-overlay combined with frequency hopping in GSM," in Proc. 8th IEEE International Symposium on Personal, Indoor and Mobile Radio Communications (PIMRC 1997), vol. 2, Helsinki, Finland, September 1997, pp. 376-380.

[15] "Mobile WiMAX - Part I: A technical overview and performance evaluation," WiMAX Forum, Tech. Rep., February 2006.

[16] T. Bonald, S. Borst, and A. Proutière, "Inter-cell scheduling in wireless data networks," in Proc. European Wireless (EW 2005), Nicosia, Cyprus, April 2005, pp. 566-572.

[17] — "Inter-cell coordination in wireless data networks," European Transactions on Telecommunications, vol. 17, no. 3, pp. 303-312, May 2006.

[18] S. Liu and J. Virtamo, "Inter-cell coordination with inhomogeneous traffic distribution," in Proc. 2nd Conference on Next Generation Internet Design and Engineering (NGI 06), València, Spain, April 2006.

[19] M. Sternad, T. Ottosson, A. Ahlen, and A. Svensson, "Attaining both coverage and high spectral efficiency with adaptive OFDM downlinks," in Proc. 58th IEEE Vehicular Technology Conference (VTC 2003-Fall), vol. 4, Orlando, FL, USA, October 2003, pp. 2486-2490.

[20] M. C. Necker, "Local interference coordination in cellular 802.16e networks," in Proc. 66th Vehicular Technology Conference (VTC 2007Fall), Baltimore, MA, USA, October 2007

[21] 3GPP TSG RAN WG1\#41 R1-050407, "Interference coordination in new OFDM DL air interface," Alcatel, Athens, Greece, Tech. Rep., May 2005.
[22] 3GPP TSG RAN WG1\#49bis R1-073187, "Interference coordination framework with results," Alcatel-Lucent, Orlando, FL, USA, Tech. Rep., June 2007.

[23] 3GPP TSG RAN WG1\#53 R1-081873, "Semi-static interference coordination method," Alcatel-Lucent, Kansas City, MO, USA, Tech. Rep., May 2008.

[24] 3GPP TSG RAN WG1\#44bis R1-060905, "Adaptive fractional frequency reuse," Nortel, Athens, Greece, Tech. Rep., March 2006.

[25] 3GPP TSG RAN WG1 LTE Ad Hoc R1-061852, "Adaptive fractional frequency reuse,” Nortel, Cannes, France, Tech. Rep., June 2006.

[26] 3GPP TSG RAN WG1 Ad Hoc R1-062150, "Adaptive fractional frequency reuse," Nortel, Tallinn, Estonia, Tech. Rep., August 2006.

[27] 3GPP TSG RAN WG1\#42bis R1-051059, "Inter-cell interference mititgation for EUTRA," Texas Instruments, San Diego, CA, USA, Tech. Rep., October 2005.

[28] 3GPP TSG RAN WG1\#44 R1-060369, "Signaling requirements to support semi-static frequency planning for inter-cell interference mitigation in EUTRA downlink," Texas Instruments, Denver, CO, USA, Tech. Rep., February 2006.

[29] M. Rahman and H. Yanikomeroglu, "Interference avoidance through dynamic downlink OFDMA subchannel allocation using intercell coordination," in Proc. 67th IEEE Vehicular Technology Conference (VTC 2008-Spring), Singapore City, Singapore, May 2008, pp. 1630-1635.

[30] G. Li and H. Liu, "Downlink dynamic resource allocation for multi-cell OFDMA system," in Proc. 58th IEEE Vehicular Technology Conference (VTC 2003-Fall), vol. 3, Orlando, FL, USA, 2003, pp. 1698-1702.

[31] R. Vilzmann, C. Bettstetter, and C. Hartmann, "On the impact of beamforming on interference in wireless mesh networks," in Proc. IEEE Workshop on Wireless Mesh Networks, Santa Clara, CA, USA September 2005.

[32] R. Ramaswami and K. Parhi, "Distributed scheduling of broadcasts in a radio network," in Proc. 8th Annual Joint Conference of the IEEE Computer and Communications Societies (INFOCOM 1989), vol. 2, Ottawa, ON, Canada, April 1989, pp. 497-504.

[33] K. Jain, J. Padhye, V. N. Padmanabhan, and L. Qiu, "Impact of interference on multi-hop wireless network performance," in Proc. 9th Annual International Conference on Mobile Computing and Networking, San Diego, CA, USA, 2003, pp. 66-80.

[34] Y. Wu, Q. Zhang, W. Zhu, and S.-Y. Kung, "Bounding the power rate function of wireless ad hoc networks," in Proc. 24th Annual Joint Conference of the IEEE Computer and Communications Societies (INFOCOM 2005), vol. 1, Miami, FL, USA, March 2005, pp. 584-595.

[35] P. Stuedi and G. Alonso, "Computing throughput capacity for realistic wireless multihop networks," in Proc. 9th ACM/IEEE International Symposium on Modeling, Analysis and Simulation of Wireless and Mobile Systems (MSWiM 2006), Torremolinos, Malaga, Spain, October 2006, pp. 191-198.

[36] 3GPP TS 25.814, Physical layer aspects for evolved Universal Terrestrial Radio Access (UTRA) (Release 7), 3rd Generation Partnership Project, June 2006.

[37] "NGMN performance evaluation methodology," Next Generation Mobile Networks Alliance," version 1.2, June 2007.

[38] "WiMAX system evaluation methodology," WiMAX Forum," version 1.0, January 2007.

[39] IEEE 802.16e, IEEE Standard for Local and metropolitan area networks, Part 16: Air Interface for Fixed and Mobile Broadband Wireless Access Systems, Amendment 2: Physical and Medium Access Control Layers for Combined Fixed and Mobile Operation in Licensed Bands, February 2006.

[40] M. C. Necker, "Local interference coordination in cellular OFDMA networks," in Proc. 12th VDE/ITG Mobilfunktagung, Osnabrück, Germany, May 2007.

[41] D. Brélaz, "New methods to color the vertices of a graph," Communications of the ACM, vol. 22, no. 4, pp. 251-256, April 1979.

[42] A. Hertz and D. de Werra, "Using tabu search techniques for graph coloring," Computing, vol. 39, no. 4, pp. 345-351, December 1987. 